\title{
ARTICLE
}

\section{Electrochemical Neutrino Detector and Its Signal Generation Scenario in Relation to Oxygen Production}

\author{
Kenji ISHIBASHI ${ }^{1 *}$, LIU Wei ${ }^{1+}$, Md. Abbul HALIM ${ }^{1++}$, Norichika TERAO $^{1}$, Hidehiko ARIMA ${ }^{1}$, \\ Yoshiaki KATANO ${ }^{2}$, and Takashi IIJIMA ${ }^{2}$ \\ ${ }^{1}$ Department of Applied Quantum Physics and Nuclear Engineering, Kyushu University, Motooka, Fukuoka 819-0395, Japan \\ ${ }^{2}$ FUGEN Decommissioning Engineering Center, Japan Atomic Energy Agency, Myojin-machi, Tsuruga 914-8510, Japan \\ ${ }^{+}$On leave to Shanghai Institute of Applied Physics, Chinese Academy of Sciences, Jialuo Road, Jiading, Shanghai 201800, P.R.China \\ ${ }^{++}$On leave to RNPD, INST, Atomic Energy Research Establishment, Ganakbari, DEPZ, Savar, Dhaka, Bangladesh
}

\begin{abstract}
A small electrochemical apparatus consisting of two electrodes and raw silk generated a considerable electric current of several tens of $\mathrm{nA}$ in the environmental condition. The output current increased with nuclear-reactor neutrino irradiation in two types of experiments. These results indicated that the detector is sensitive to environmental and reactor neutrinos. Additional experimental studies were carried out on oxygen-concentration variation and conducting-wire-material dependence. These experimental data suggest that the output signal is followed by generation of oxygen and is related to weak-interaction products. We infer that neutrinos have a mass-generation mechanism which is affected by a scalar auxiliary field. When a mass consistency to self energy is disturbed by an external application of the scalar auxiliary field, a neutrino may break up into two groups of fragments, which work to drive current flow to produce the output signal. The biological product of raw silk is supposed to retain the neutrino fragments therein and produce the scalar auxiliary field.
\end{abstract}

\section{KEYWORDS: neutrino, electrochemical, detector, nuclear reactor, oxygen, weak interaction}

\section{Introduction}

Neutrinos make only the weak interaction. The quite small interaction cross section of neutrinos was successfully explained by the electroweak theory. ${ }^{1)}$ For low-energy solar neutrinos, measurements were carried out by radiochemical experiments such as SAGE, ${ }^{2)}$ where the neutrino detection threshold was $0.23 \mathrm{MeV}$. Neutrinos with energies below this energy have not been measured for far.

It is known well that determination of electromagnetic potentials requires constraint conditions like Landau and Fermi gauges. ${ }^{3)}$ Such gauges accompany a special auxiliary field $^{4)}\left(B^{0}\right.$ thereafter), except the Landau gauge giving Lorentz condition of $\sum\left(\partial / \partial x^{\mu}\right) A^{\mu}=0$ for four-dimensional potentials $A^{\mu}$. The auxiliary field $B^{0}$ possesses an energy density like electric and magnetic fields. When considerably low energy neutrinos receive certain strength of external field such as auxiliary field $B^{0}$, it may disturb the mass-generation mechanism of neutrino. Although the mechanism is not elucidated, the external auxiliary field may give rise to influence to the mass state. It is interesting to search materials that produce $B^{0}$ and influence the weak interaction of neutrinos.

We may have found the material that possibly generates the external fields and promotes the weak-interaction reaction. An electrochemical apparatus ${ }^{5)}$ was developed which was supposedly sensitive to low energy neutrinos. Experiments were performed under irradiation of both environmental and nuclear reactor neutrinos. Further experiments were attempted to be carried out in this work to study the signal generation scenario.

*Corresponding Author, E-mail: kisibasi@nucl.Kyushu-u.ac.jp

\section{Basic Experiments \\ 2.1 Apparatus}

Figure 1 illustrates the cross section of the experimental apparatus. ${ }^{5)}$ The experimental apparatus was made in a Teflon container with a volume of about $100 \mathrm{ml}$. The Teflon container had purified water of $50 \mathrm{ml}$ in the lower half region, where gold and glassy carbon plates were utilized as electrodes. Both plates were $20 \times 50 \mathrm{~mm}$ in dimension. The thickness was $0.1 \mathrm{~mm}$ for the gold plate, and $1.0 \mathrm{~mm}$ for the glassy carbon one. Electrodes were boiled in nitric acid for cleaning the surface, and subsequently rinsed by ultrasonic wash in purified water. Before pouring in the apparatus,

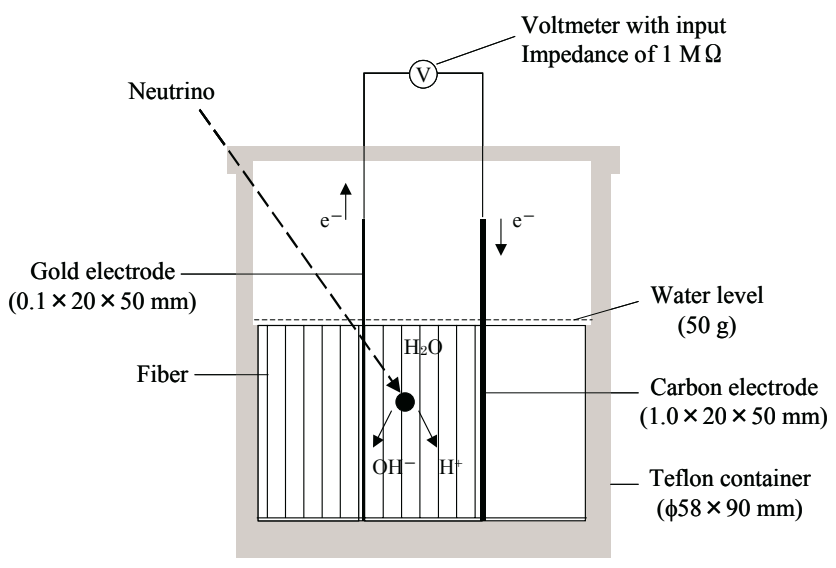

Fig. 1 Schematic view of the experimental apparatus. Gold and glassy carbon electrodes are immersed in purified water in a Teflon container. Raw silk fiber is placed around the gold electrode. The current between the two electrodes is measured by a voltmeter with an input impedance of $1 \mathrm{M} \Omega$. 


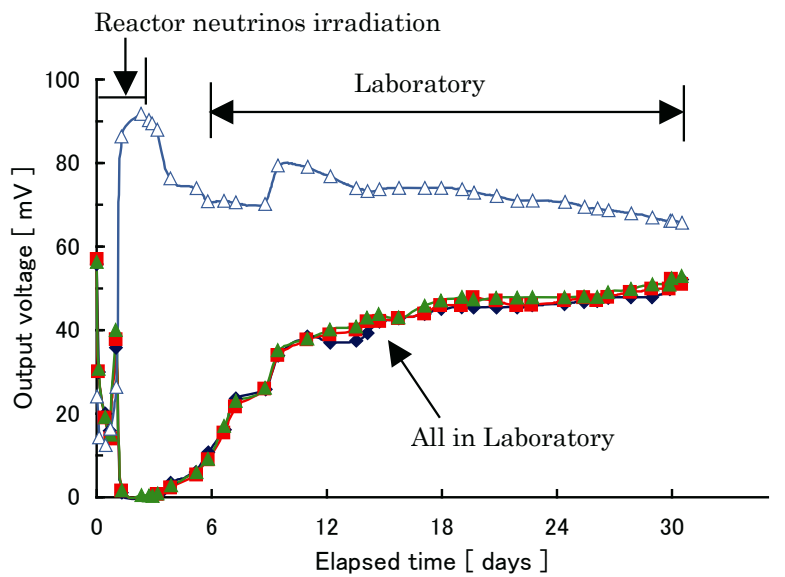

Fig. 2 Response of the apparatus to reactor and environmental neutrinos. The effect of reactorneutrino irradiation clearly appeared in the output voltage in the open triangles.

purified water was bubbled by nitrogen gas for reducing oxygen dissolved. Raw silk is the biological product that is produced from silkworms. Raw silk fibers of $0.5 \mathrm{~g}$ in weight were set onto both sides of the gold plate, i.e., total $1.0 \mathrm{~g}$. Silk fibers of $17 \mathrm{~mm}$ long were used in most cases. However, short-cut fiber of few mm was adopted in the last experiment because of easy assembling of the apparatus. Use of short-cut silk tended to lead to a larger voltage in the initial period but the equilibrium values are mostly the same as in the longer fiber case. A voltmeter with an input impedance adjusted to 1 $\mathrm{M} \Omega$ was connected by copper wires between the two electrodes. The experimental apparatus was placed in a temperature-controlled incubator at $27^{\circ} \mathrm{C}(300 \mathrm{~K})$.

\subsection{Results for reactor and environmental neutrinos}

Nuclear reactors are capable of producing an intense flux of neutrinos. They are mostly electron antineutrinos from fission fragments, and include a small amount of normal neutrinos from products generated through neutron reactions such as $(n, 2 n)$. The above experiment was performed under reactor-neutrino irradiation at the FUGEN nuclear power station, which is a type of thermal-neutron reactor with an electric power of $165 \mathrm{MW}$. The entire apparatus was set at a position $33.5 \mathrm{~m}$ apart from the reactor core. The experimental position was outside the container vessel in a non-radiation controlled area, where neutrons and gamma rays were well shielded at a conventional environmental dose-rate level of about $0.1 \mu \mathrm{Sv} / \mathrm{h}$. The irradiation produced a reactor neutrino flux of $7.4 \times 10^{11} \mathrm{~cm}^{-2} \mathrm{~s}^{-1}$. The irradiation was made for three days. After the irradiation, the apparatus was carried back to our laboratory at Kyushu University.

The results are shown by open triangular marks in the upper region of Fig. 2. Simultaneously, the experiment in environmental condition was also performed all time at Kyushu University, and the output signals are presented by the lower three curves (solid marks) in the same figure. The three results agree with one another. For the upper curve by reactor-neutrino irradiation, a considerable increase of output signal is seen in the first three days, and should be attributed

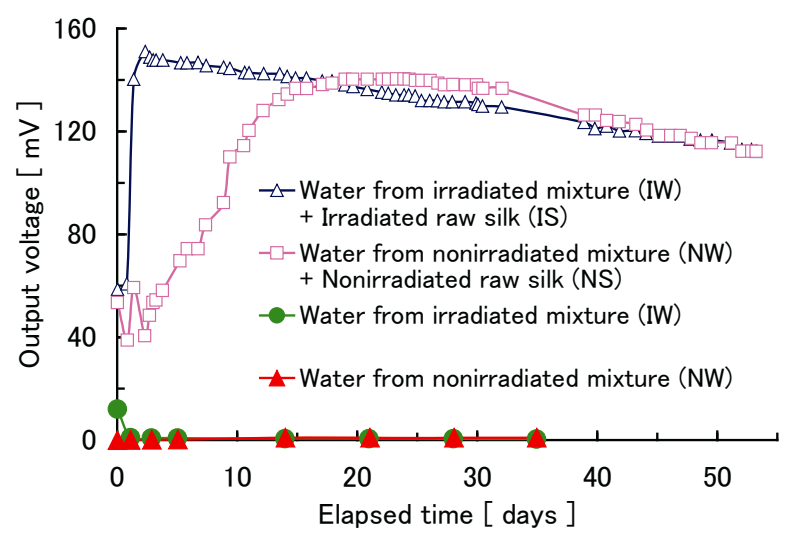

Fig. 3 Effect of reactor-neutrino irradiated raw silk on the output voltage of experimental apparatus.

to the intense neutrino flux from the nuclear reactor. This suggests that neutrinos from the nuclear reactor interact with the mixture of purified water and the raw silk. Since only 50 $\mathrm{g}$ of water was used for interacting with reactor neutrinos, these results are not attributed to the ordinary weak interaction with typical cross section of $10^{-40} \mathrm{~cm}^{2}$. Kyushu University is about $50 \mathrm{~km}$ from the nearest nuclear power station, so that the influence of reactor neutrinos on the output voltage of this apparatus could be ignored at our laboratory. The output voltage, however, was still higher after returning back to our laboratory. Some materials or particles are supposed to be generated in the apparatus.

\subsection{Another reactor-neutrino effect}

Neutrino effects were studied with an alternative approach. A mixture was prepared containing purified-water and raw-silk: purified water of $800 \mathrm{~g}$ was poured in a 1-liter glass bottle, after raw silk of $100 \mathrm{~g}$ was placed. Two bottles were prepared at the same time. One of the bottles was irradiated with the reactor neutrinos with a flux of $6.35 \times 10^{12} \mathrm{~cm}^{-2} \mathrm{~s}^{-1}$ for three days. After being brought back to Kyushu University, the water was taken out from this bottle. It was designated as irradiated water (IW). The other bottle was kept at all times at our laboratory of Kyushu University, and the water from this bottle was designated as non- irradiated water (NW). In addition, two kinds of raw silk were prepared. One was irradiated with the reactor neutrinos with the same flux for three days (irradiated raw silk; IS), while the other was stored in our laboratory (nonirradiated raw silk; NS). After one month storage, the apparatuses were assembled with these materials at our laboratory.

In Fig. 3, the results of output voltage by the use of either nonirradiated (NW) or irradiated water (IW) alone are shown by solid marks in the lowest region. One can see that the experiments by either NW or IW without raw silk produced basically no signal. Open-rectangular data for nonirradiated water and $1 \mathrm{~g}$ silk $(\mathrm{NW}+\mathrm{NS})$ gradually increase from initial $50 \mathrm{mV}$ to $140 \mathrm{mV}$ in two weeks. The increasing behavior is somewhat similar to that of the solid marks in Fig. 2 for the except the signal offset. In contrast, open-triangular marks for irradiated water and $1 \mathrm{~g}$ silk (IW+IS) reached $150 \mathrm{mV}$ in a 


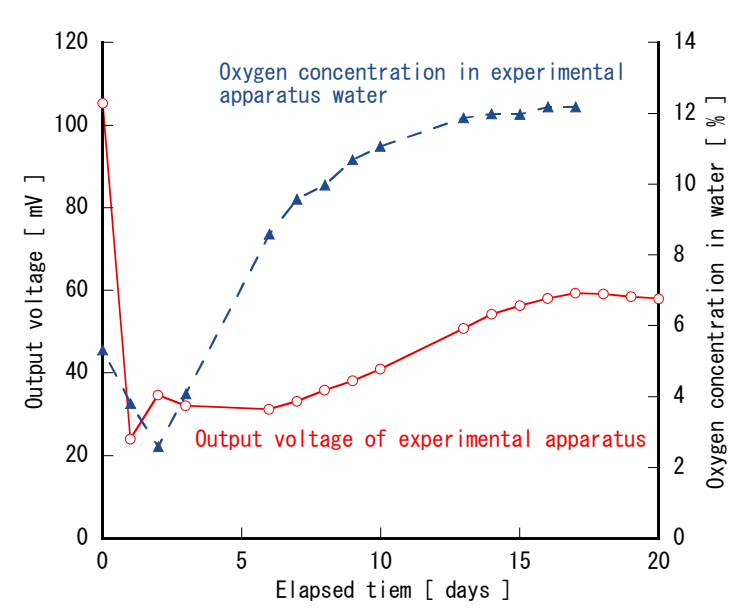

Fig. 4 Oxygen concentration measured in the experimental apparatus in environmental condition.

few days. From the difference of data between NW+NS and IW+IS in Fig. 3 as well as that between two curves in Fig. 2, it is clear that the reactor neutrinos clearly influence the output voltage of the apparatus. This fact suggests that the output signal shown in the lower part of Figs. 2 and 3 should originate from the interaction with environmental neutrinos.

\section{Additional Experiments on Signal Generation}

For studying the signal generation mechanism, two additional experiments were carried out.

\subsection{Oxygen concentration}

The oxygen concentration was measured with a portable dissolved oxygen meter (Mettler Tredo, MO-128). The cylindrical sensor was directly inserted to water in the Teflon container. An $\mathrm{O}$ ring seal was set around the cylinder to prevent the air from flowing. The results are shown in Fig. 4. The open circles stand for the output voltage of the apparatus with the impedance of $1 \mathrm{M} \Omega$. The solid triangles indicate the oxygen concentration in water. The oxygen concentration decreases for initial two days, and subsequently gradually glow to reach $12 \%$ in almost two weeks.

\subsection{Use of short-cut fiber and wire-material dependence}

Use of short-cut silk fiber was adopted for ease of fiber-packing work. The output voltage is shown in Fig. 5 with solid triangles for copper wire. The initial peak is enhanced in 2 days. The equilibrium voltage after two weeks is almost the same as the previous values of solid marks in Fig. 2. The output voltage was also measured by the use of conducting wires with different metallic materials such as $\mathrm{Au}$, $\mathrm{Ag}, \mathrm{Fe}$ and $\mathrm{Zn}$. The electric resistance of these wires is of the order of $1 \mathrm{~m} \Omega$, while the input impedance of the voltage meter is as large as $1 \mathrm{M} \Omega$. Since the conducting wires were attached on the top of electrodes in Fig. 1, they were not immersed in the water. The output signals are higher in $\mathrm{Au}$ and Ag, while they are lower in Fe and Zn particularly before one weak. The use of the electromagnetic Ohm's law should lead to the simple result that the output voltage is independent on the conducting wire materials within an experimental error. However, an appreciable difference was seen on the voltage

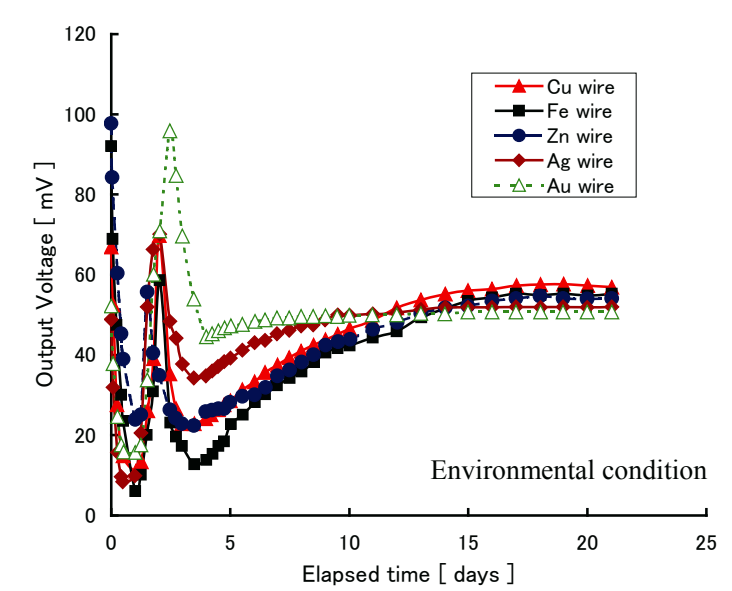

Fig. 5 Output voltage of the electrochemical detector by the use of different conducting wire materials. Short-cut silk fiber of few mm was used.

behavior. The results suggest that the conducting electrons in wire may be followed by some particles that are influenced by the conducting materials.

\section{Signal Generation Scenario}

It seems to be difficult to explain the experimental results. The difficulty forced us to make two suppositions: (1) Neutrino is constituted by two groups of constituents, $\xi_{Q}$ and $\xi_{Q d}$, where the group $\xi_{Q}$ has a weak charge $Q$ and that of $\xi_{Q d}$ owns a weak dipole moment $Q_{d}$. In fact, the internal weak magnetic fields are well cancelled out, ${ }^{6)}$ only when the neutrino flies with left-rotated orbital rotation of $l_{z}=-1$ with a velocity close to the light velocity. A relativistic consideration $^{6)}$ may give the view that the candidate of $Q$-type constituent is a particle related to gamma matrix $\gamma_{1}$, while that of $Q_{d}$-type includes three particles 0,2 and 3 related to matrices $\gamma_{0}, \gamma_{2}$ and $\gamma_{3}$. (2) The mass of neutrino constituent is formulated by $\mathrm{V}$ - and AV-type kinetic momentums with magnetic field and the AV-type auxiliary field $B^{0}$. Then, the kinetic mass of particle $v$ in the $\xi_{Q d}$ group is expressed by

$$
m_{v}^{k i n}=\sqrt{\begin{array}{l}
\sum_{\mu=0 \sim 3}\left(p_{\mu v V}-Q_{d v} B_{\mu v V}\right)^{2} \\
-\sum_{\mu=0 \sim 3}\left(p_{\mu v A}-Q_{d v} B_{\mu v A}\right)^{2}+\left(Q_{d v} B_{v A}^{0}\right)^{2}
\end{array}}
$$

where $\mu$ indicates directions of $c t, x, y$ and $z$ and $v$ the particle number. The kinetic mass is constituted by difference between $\mathrm{V}$ - and AV-momentum terms and the energy of the AV-type auxiliary field $B^{0}$. The kinetic motion takes place so that the kinetic mass may always be equal to the self-mass that is determined by individual self potential field. ${ }^{6)}$

Suppose that a low-energy neutrino $v=\xi_{Q} \xi_{Q d}$ receives an external AV-type auxiliary field $B^{0}$ of considerable strength. The external field $B^{0}$ readily increases the kinetic mass. This may bring the inconsistency between kinetic mass and self-mass, and the neutrino may break up into two fragments of $\xi_{Q}$ and $\xi_{Q d}$. Each fragment may require to be attached to 
another partner to gain a mass base. It is inferred that the force between two fragments are repulsive at rest condition. ${ }^{6)}$ The fragment $\xi_{Q}$ efficiently creates the auxiliary field. The biological product of raw silk may contain the accumulated fragments $\xi_{Q}$ to generate the auxiliary field $B^{0}$.

From the above view, a signal generation scenario is considered as follows. The raw silk fiber originally accumulates some amount of $\xi_{Q}$ therein and to produce the AV-type auxiliary field $B^{0}$. Due to the external $B^{0}$ from the raw silk in the apparatus, a neutrino $v\left(=\xi_{Q} \xi_{Q d}\right)$ may separate into the two fragments $\xi_{Q}+\xi_{Q d}$, breaking up a water molecule into $\mathrm{OH}^{-} \xi_{Q d}$ and $\mathrm{H}^{+} \xi_{Q}$ ions as

$$
\xi_{Q} \xi_{Q d}+\mathrm{H}_{2} \mathrm{O} \rightarrow \mathrm{OH}^{-} \xi_{Q d}+\mathrm{H}^{+} \xi_{Q} .
$$

For simplicity, only ions were indicated in Fig. 1. The $\mathrm{OH}^{-} \xi_{Q d}$ ions move to the gold electrode, to which electron- $\xi_{Q d}$ pairs in spin coupling are transferred. A recombination of two $\mathrm{OH}^{-}$may make water, oxygen and electron- $\xi_{O d}$ pairs as

$$
2 \mathrm{OH}^{-} \xi_{Q d} \rightarrow \mathrm{H}_{2} \mathrm{O}+(1 / 2) \mathrm{O}_{2}+2 \mathrm{e}^{-} \xi_{Q d} \text {. (gold electrode) }
$$

If $\xi_{Q d}$ and $\xi_{Q}$ are not attached to individual ions unlike the right-hand side of eq. (1), $\mathrm{OH}^{-}$should quickly recombine with $\mathrm{H}^{+}$to return to a water molecule: it is impossible for the created $\mathrm{OH}^{-}$to reach the gold electrode. The $\mathrm{e}^{-} \xi_{Q d}$ pairs conduct through a wire to arrive at the glassy-carbon electrode. Since the auxiliary field $B^{0}$ works like a scalar potential for fragments $\xi_{Q d}$, the difference of $B^{0}$ between electrodes is considered to contribute to the flow of $\mathrm{e}^{-} \xi_{Q d}$ in conductor. The conduction of $\mathrm{e}^{-} \xi_{Q d}$ is supposed to be dependent on the wire materials due to existence of $\xi_{Q d}$. Meanwhile, the $\mathrm{H}^{+} \xi_{Q}$ ions diffuse toward the glassy-carbon electrode, combine with oxygen molecules, and absorb electrons to form water molecules as

$$
2 \mathrm{H}^{+} \xi_{Q}+(1 / 2) \mathrm{O}_{2}+2 \mathrm{e}^{-} \rightarrow 2 \mathrm{H}_{2} \xi_{Q} \text { O.(carbon electrode)(3) }
$$

A current is thus induced in the circuit between the electrodes. Consequently, the output voltage of the apparatus experimentally appears over the input impedance. During the diffusion of $\mathrm{O}_{2}$ from the gold to glassy-carbon electrodes, in principle, no voltage signal is generated in spite of continuous accumulation of $\mathrm{OH}^{-} \xi_{Q d}$ and $\mathrm{H}^{+} \xi_{Q}$ by eq. (1); the reaction of eq. (2) takes place only by initially dissolved oxygen.

\section{Discussion about the experimental output- voltage behavior}

From the reaction equations of (1) to (3), we consider the reason for the experimental output-voltage behavior. For the experimental data by the use of raw silk, initial peaks appeared around 1.5-2 days in Figs. 2-5 (appreciable in Fig. $5)$ and they disappeared quickly. It seems to take about one day for $\mathrm{OH}^{-} \xi_{Q d}$ to be accumulated by eq. (1) and to diffuse toward the gold electrode. Following the reaction of eq. (2), oxygen molecules $\mathrm{O}_{2}$ are generated and accumulated and they should diffuse toward the carbon electrode. The accumulation and diffusion of $\mathrm{O}_{2}$ seem to be insufficient during few days. Accordingly the output voltage appears by the reaction between initial $\mathrm{H}^{+}$and $\mathrm{O}_{2}$ remaining at the early stage ( 1.5 days) in the apparatus, but it disappears soon after consumption of $\mathrm{H}^{+}$and $\mathrm{O}_{2}$. In fact, Fig. 4 indicates that it took about 10 days for $\mathrm{O}_{2}$ to be accumulated and equilibriumly diffuse toward the carbon electrode. The oxygen gas increase is followed by accumulation of $\xi_{Q}$ in water through eq. (3). The oxygen accumulation and diffusion are understood to be the rate-determining process for charge conduction for the output voltage.

\section{Conclusion}

An electrochemical apparatus consisting of two electrodes and raw silk generated a considerable electric current of several tens of $\mathrm{nA}$ in the environmental condition. The output voltage increased with nuclear-reactor neutrino irradiation. The experimental results suggested that the output voltage came from the interaction of incident neutrinos. As one of plausible explanations for the neutrino reaction, the hypothesis was proposed that the existence of the external auxiliary field may readily break low-energy neutrinos and produce an electric signal through the functions of dissociated water molecules through assistance of neutrino fragments. Accumulation and diffusion of oxygen gas as well as neutrino fragments were considered to be the rate-determining process for the voltage increase for environmental neutrinos.

\section{Acknowledgements}

The authors wish to express their gratitude to staff of FUGEN Nuclear Power Station.

\section{Reference}

1) S.L. Glashow, "The status of the electroweak sector of the standard model," Nucl. Phys. 22 579-588(1961), A. Salam, "Weak and Electromagnetic Interactions," Elementary Particle Theory (ed. By N. Svartholom, Almquist and Forlag. 1968), 367-377, S. Weinberg, “A Model of Leptons," Phys. Rev. Letters, 19 1264-1266 (1967).

2) J.N. Abdurashitov, et al., "Measurement of the solar neutrino capture rate with gallium metal," Phys. Rev. C 60 558011-5580132 (1999).

3) G. Kallen, Quantum Electrodynnamics, Springer-Verlag, Berlin (1972).

4) R. Utiyama, "Theory of Invariant Variation and the Generalized Canonical Dynamics," Prog. Theor. Phys. Suppl. 9, 19-44 (1959).

5) Liu Wei, K. Ishibashi, H. Arima, Y. Katano, Y. Naoi, "Possible Detection of Natural Neutrinos by Use of Small Apparatus," $J$. Nucl. Sci. Technol., Suppl. 4, 487-490 (2004).

6) K. Ishibashi, et al., "Neutrino Properties and Their Usefulness Suggested by Recent Electrochemical Neutrino Detection Results," presented at this symposium. 